Le festivalier: lecteur, auteur et créateur de sens

\title{
Mylène Fréchette
}

\section{Résumé}

Le festival littéraire Les Correspondances d'Eastman, qui a lieu en Estrie, au Québec, offre une programmation variée comprenant notamment des lectures publiques, des entrevues d'auteurs, des spectacles littéraires et des ateliers d'écriture. Sa spécificité consiste à proposer aux visiteurs d'écrire des lettres dans des jardins d'écriture aménagés par les citoyens du village d'Eastman. D’un côté, en incitant l'ensemble des visiteurs à prendre la plume, le festival encourage la démocratisation de la littérature et de la création littéraire. D'un autre côté, le festival supporte la professionnalisation des écrivains reconnus en rémunérant leur participation aux activités et en leur offrant une plateforme de promotion et une visibilité accrue. À partir des tensions sous-jacentes à ces deux facettes du festival, l'article propose d'explorer le.s rôle.s des festivaliers dans le cadre des Correspondances d'Eastman et de le.s comparer avec celui des écrivains professionnels. L'analyse sera particulièrement centrée sur les interactions entre les visiteurs et les écrivains professionnels, qui oscillent constamment entre la proximité, l'intimité et la distance.

\section{Abstract}

Les Correspondances d'Eastman, a literary festival held in the Estrie region of Quebec, offers a varied program that includes public readings, author interviews, literary performances, and writing workshops. The festival specifically aims to offer visitors the opportunity to write letters in writing gardens laid out by the citizens of Eastman village. On the one hand, by encouraging all visitors to write, the festival encourages the democratization of literature and creative writing. On the other hand, the festival supports the professionalization of recognized writers by compensating them for their participation in activities and offering them a platform to promote their works and increase their visibility. Based on the tensions underlying these two 
aspects of the festival, this article aims to explore the roles festivalgoers play in Les Correspondances d'Eastman and to compare them with those of professional writers. The analysis will focus primarily on the interactions between visitors and professional writers, which constantly oscillate between proximity, intimacy, and distance.

Les festivals littéraires sont une forme de médiatisation de la littérature assez récente, mais dont la popularité croît d'année en année. Apparus au milieu du $\mathrm{XX}^{\mathrm{e}}$ siècle dans l'univers littéraire anglo-saxon ${ }^{\mathrm{I}}$, les festivals littéraires ont émergé plutôt tardivement dans le monde francophone, soit au tournant des années 1980, puis se sont multipliés dans les dernières années. Le Québec ne fait pas exception à cette nouvelle vogue. On y retrouve par exemple le Festival international de la littérature et le Festival international Metropolis Bleu, à Montréal, et le festival Québec en toutes lettres, à Québec. C'est également le Québec qui accueille le «seul festival littéraire estival en Amérique du Nord $^{2}$ », c'est-à-dire Les Correspondances d'Eastman, une réunion ayant lieu chaque année au début du mois d'août à Eastman, un village des Cantons de l'Est. Créé en 2003 par les écrivains Jacques Allard, Louise Portal et Nicole Fontaine, accompagnés de Philippe-Denis Richard, avocat, et Line Richer, première directrice générale de l'organisme, ce festival a été inspiré d'un modèle français, Les Correspondances de Manosque, lui-même fondé en 1999. Les premiers objectifs de la mise sur pied d'un tel festival étaient de "donner le goût d'écrire et de lire " " au plus grand nombre possible, de «valoriser le livre et le travail des écrivains ${ }^{4}$ » et de célébrer toutes les facettes de la littérature. En plus de proposer des lectures publiques et des entrevues d'auteurs, le festival promeut un accès généralisé à la création littéraire et, plus largement, à l'écriture, en incitant les visiteurs à écrire des lettres dans des jardins spécialement aménagés à cet effet.

Comme ils sont à la fois les récepteurs des œuvres écrites par des écrivains professionnels et les créateurs de leurs propres textes, les

I Le plus ancien festival littéraire, le Cheltenham Literature Festival, situé en Angleterre, a été fondé en 1949. Il est toujours actif de nos jours.

2 François Lévesque, "Dix ans de correspondances à Eastman. Le festival Les Correspondances d'Eastman ouvre ses jardins aux passionnés de lettres", Le Devoir, 4 août 20I2. http://www.ledevoir.com/culture/livres/356014/dix-ansde-correspondances-a-eastman.

3 Les Correspondances d'Eastman. http://www.lescorrespondances.ca/a-propos.

4 Ibid. 
festivaliers endossent un rôle complexe et multiple dans le cadre des Correspondances d'Eastman. Les différentes activités auxquelles ils prennent part élargissent leur fonction, qui n'est pas cantonnée à celle d'un simple lecteur. Loin d'être relégués à une position subalterne, ils évoluent parmi les acteurs du milieu du livre, par exemple en rencontrant les écrivains lors des séances de dédicaces et en posant des questions pendant les entrevues. Je propose donc d'interroger la place qu'occupent les festivaliers à l'intérieur des Correspondances d'Eastman par rapport aux autres participants, que ce soient les écrivains, les éditeurs ou les animateurs. Comment s'organise la participation des festivaliers aux différentes activités? En quoi leurs interventions infléchissent-elles les échanges? Quels rapports entretiennent-ils avec les acteurs du monde du livre? L'étude des interactions entre les festivaliers et les professionnels du livre au sein du festival permettra de mieux cerner les rôles endossés par chaque catégorie de participants durant les festivités.

Afin de dresser un portrait des visiteurs, je m'appuierai sur mes observations personnelles, réalisées au cours des trois dernières éditions du festival en 20I7, 2018 et 2019, ainsi que sur les résultats d'un sondage mené directement sur le site des Correspondances d'Eastman en 20I9. Entre le jeudi 8 août 2019 et le dimanche II août 20I9, 74 festivaliers 5 ont rempli un questionnaire rassemblant 3I questions à choix multiples ou à réponse courte, réparties en 4 sections: I) Informations sociodémographiques (âge, sexe, niveau de diplôme, catégorie socioprofessionnelle, revenu annuel et situation matrimoniale) ; 2) Enfants et famille (âge des enfants, activités du volet jeunesse); 3) Habitudes culturelles (pratiques de lecture et d'écriture, sorties culturelles, etc.); 4) Expériences aux Correspondances d'Eastman (activités favorites, attraits du festival, rapports avec les écrivains invités, appréciation de l'expérience, etc.). Les répondants du sondage constituent un échantillon empirique somme toute faible, qui ne prétend pas à la représentativité des festivaliers des Correspondances d'Eastman. La plupart des personnes sondées témoignaient d'une grande ouverture envers l'organisation et d'une appréciation fortement positive du festival, ce qui aurait pu biaiser les données récoltées. Celles-ci présentent tout de même un grand nombre de similarités avec les résultats des études de plus grande

34 questionnaires ont été remplis avant ou après un café littéraire ou une entrevue, I6 dans un jardin d'écriture, I6 avant un spectacle du soir, 5 dans l'espace jeunesse et 3 dans un espace intermédiaire entre différents sites. 
envergure sur les publics des festivals ${ }^{6}$, ce qui laisse croire que les résultats du sondage sont fiables. Pour compléter le portrait du festival et de ses participants, je mettrai également à profit mes discussions informelles avec des bénévoles, des festivaliers et des auteurs invités, ainsi que les entrevues menées auprès du directeur général du festival, Raphaël Bédard-Chartrand, et de la porte-parole de la $16^{\mathrm{e}}$ édition (2018), Stéphanie Boulay ${ }^{7}$.

\section{Vers une démocratisation de la littérature}

Raphaël Bédard-Chartrand, directeur général de l'organisme depuis 20I4, présente Les Correspondances d'Eastman comme un événement qui vise à décloisonner la littérature en la jumelant avec d'autres arts et en la rendant accessible à un plus large public. Le festival s'inscrit en ce sens dans une volonté de démocratisation de la culture. Cela s'observe notamment dans la programmation du festival, qui présente sur un pied d'égalité les livres populaires, la littérature jeunesse et les écrits savants. Par exemple, la $16^{\mathrm{e}}$ édition des Correspondances d'Eastman mettait à l'honneur l'essayiste, romancier et professeur Yvon Rivard, qui était l'invité de la grande entrevue ${ }^{8}$ du vendredi Io août 20I8. Le programme présentait l'activité comme suit: «Étalée sur plus de quarante années d'écriture de romans et d'essais, l'œuvre d'Yvon Rivard tente de penser l'exigence de vivre au-delà du désir et au cœur même de la prose des jours ${ }^{9}$." Comme le laisse penser cette courte description, l'entrevue s'adressait à un public lettré, intéressé par les questions liées à la création littéraire et à la philosophie. La discussion était animée par Étienne Beaulieu, directeur de la programmation des Correspondances d'Eastman et professeur de littérature au Cégep

6 Voir notamment Gisèle Sapiro (dir.), L'écrivain.e à la rencontre de son public. Enquête sur le public du festival Les Correspondances de Manosque, Paris, Centre européen de sociologie et de science politique, 20I2, 38 p.; Emmanuel Négrier, Aurélien Djakouane et Marie Jourda, Les publics des festivals, Paris, Éditions Michel de Maule/France Festivals, 20IO, 287 p.; Wenche Ommundsen, «Literary Festivals and Cultural Consumption", Australian Literary Studies, vol. 24, $\mathrm{n}^{\circ} \mathrm{I}$, 2009, p. 19-34.

7 Voir les guides d'entrevue en Annexe I.

8 Les grandes entrevues sont des activités qui donnent la parole à un auteur particulièrement renommé et aimé du public. Une seule grande entrevue, d'une durée approximative d'une heure, est prévue par jour.

9 Grande entrevue "Yvon Rivard», Programme du 16 e festival littéraire Les Correspondances d'Eastman, 9 au I2 août 20I8, p. 3. 
de Drummondville, qui a réalisé un doctorat en études littéraires à l'Université McGill sous la direction d'Yvon Rivard. Étant donné la formation universitaire des deux participants, les sujets plutôt abstraits abordés lors de l'entrevue ont été traités avec profondeur, à partir d'un point de vue théorique, voire savant.

Le lendemain, les festivaliers pouvaient assister à une table ronde sur le roman policier, discussion davantage axée sur la trame narrative des romans. La description de cette activité comparait les polars avec le cinéma, ce qui laisse entrevoir une tentative d'accrocher un large public non uniquement composé d'adeptes de littérature: "Entrer dans un univers de fiction comme dans un film, suspendu au rythme des phrases et des événements ${ }^{\mathrm{IO}}$." L'auteure Chrystine Brouillet, véritable vedette du polar québécois, participait à cette activité aux côtés de Martin Michaud, dont la série policière Victor Lessard a été adaptée au petit écran, et Marie-Ève Bourassa, auteure de la trilogie Red Light, qui a connu autant de succès auprès du public que de la critique.

En abordant un large éventail de sujets et de thématiques littéraires, la programmation semble favoriser le rassemblement de lecteurs de tous horizons au cœur d'une manifestation culturelle hétéroclite. Dans les dernières années, l'équipe du festival a mis sur pied plusieurs mesures visant à rajeunir la clientèle et à rejoindre de nouveaux publics. D'une part, les étudiants obtiennent un rabais de $50 \%$ sur la majorité des activités, et la littérature jeunesse dispose de sa propre section sur le site du festival, en plein centre du village. L'espace jeunesse, créé en 2013, propose des animations gratuites, telles que des lectures et des rencontres d'auteurs spécialement conçues pour les enfants et les adolescents, et comprend un espace réservé pour les jeux et les bricolages. Ce nouveau volet du festival vise à attirer de jeunes familles et à faire naître l'amour de la lecture et de l'écriture chez les plus petits.

D’autre part, le comité organisateur déploie des efforts afin de rejoindre un vaste public qui ne se limite pas à une élite lettrée. L'emplacement géographique du festival n'est sans doute pas étranger à cet objectif. Les Correspondances d'Eastman ont lieu dans un milieu régional, mais demeurent facilement accessibles pour la population des grandes villes: situé en bordure de l'autoroute Io reliant la ville de Sherbrooke à celle de Montréal, le festival se déroule

io Café littéraire "À l'intérieur du suspens", Programme du i 6 e festival littéraire Les Correspondances d'Eastman, 9 au I2 août 20I8, p. 4. 
à environ 50 kilomètres de Sherbrooke, à IIo kilomètres de Montréal et à 265 kilomètres de la ville de Québec. La majorité des activités culturelles se déroulant dans les grands centres, on peut se demander si l'implantation d'un festival littéraire à Eastman permet de rejoindre un nouveau public moins familier avec les manifestations culturelles. Les Correspondances d'Eastman favorisent-elles un premier contact entre les habitants de la région et la littérature, ou attirent-elles plutôt un public d'initiés? Deux études de provenance, basées respectivement sur 665 et 754 codes postaux, ont été réalisées en 2015 et en 2018 auprès de la clientèle des Correspondances d'Eastman. Les données, recueillies par des bénévoles sur les différents sites du festival, ont été analysées par la firme Robert Harmegnies Marketing. L'étude de provenance réalisée sur le site du festival en 2018 a révélé que près de la moitié des visiteurs $(42 \%)$ résidaient à moins de $40 \mathrm{~km}$ d'Eastman ${ }^{\text {II }}$. Par ailleurs, les résultats du sondage mené auprès des festivaliers de la $17^{\mathrm{e}}$ édition du festival (2019) montrent que $35 \%$ des visiteurs ne se considèrent pas comme des habitués de ce type d'événements ${ }^{\mathrm{I2}}$, ceux-ci fréquentant peu ou pas de manifestations littéraires en dehors des Correspondances d'Eastman. On peut supputer que, bien que les festivals littéraires - comme tout festival culturel - attirent habituellement une majorité de spectateurs lettrés disposant d'un fort capital culturel ${ }^{13}$, Les Correspondances d'Eastman parviennent à diversifier leur public en attirant tout autant des visiteurs habitués que des néophytes provenant d'un milieu à la fois régional et citadin. Ces différents types de publics sont amenés à cohabiter au sein du festival, ce qui se reflète dans les activités proposées.

La programmation offerte par Les Correspondances d'Eastman met à contribution autant les auteurs invités que les festivaliers. On y retrouve notamment des lectures publiques, des tables rondes et des entrevues d'auteurs, pendant lesquelles les visiteurs interviennent parfois de façon spontanée. Le festival propose aussi des ateliers d'écriture animés par des professionnels, au cours desquels les festivaliers expérimentent l'art d'écrire de façon amatrice. Ce qui

II Pour consulter les résultats détaillés des deux études de provenance, voir l'Annexe II.

I2 Voir le graphique en Annexe III.

I3 Gisèle Sapiro (dir.), L'écrivain.e à la rencontre de son public. Enquête sur le public du festival Les Correspondances de Manosque, Paris, Centre européen de sociologie et de science politique, 20I2, p. 9; Emmanuel Négrier, Aurélien Djakouane et Marie Jourda, Les publics des festivals, Paris, Éditions Michel de Maule/France Festivals, 20IO, p. 7 I. 
distingue Les Correspondances d'Eastman des autres événements littéraires sont les jardins d'écriture, prêtés et aménagés par les citoyens d'Eastman, dans lesquels les festivaliers sont invités à écrire des lettres. Selon Stéphanie Boulay, porte-parole de la $16^{\mathrm{e}}$ édition (20I8), cette facette du festival permet d' «aller chercher le créateur en chaque personne ${ }^{\mathrm{I} 4} »$. Par le biais de cette activité, Les Correspondances d'Eastman mettent de l'avant la figure de l'écrivain amateur, dont la pratique littéraire, pour différentes raisons, demeure confinée à la sphère domestique. Les amateurs, en effet, n'ont pas accès à l'existence publique en tant qu'écrivains autorisée par la publication d'un livre ${ }^{\mathrm{IS}}$. N'ayant publié aucun ouvrage auprès d'un éditeur reconnu, les auteurs amateurs ne disposent d'aucune reconnaissance de la part de l'institution littéraire, ce qui fait que leur identité d'écrivains n'est pas admise publiquement; ils n'existent comme écrivains que pour eux-mêmes, ou tout au plus pour un petit groupe de personnes. Par la mise en place des jardins d'écriture, le festival eastmanois agit comme un lieu privilégié pour les auteurs amateurs, étant donné qu'il leur permet d'exister publiquement en tant qu'écrivains et d'agir comme tels pendant quelques jours. Par ailleurs, Les Correspondances d'Eastman encouragent tous les festivaliers, sans exception - et pas seulement ceux qui se sentent déjà "écrivains" — à s'adonner à l'écriture, peu importe les connaissances et les aptitudes littéraires qu'ils possèdent. Dans cette optique, le festival promeut une "vision "démocratique" du don et de la vocation", selon laquelle "chacun serait doté des mêmes talents créateurs, d'un même potentiel de créativité qui ne demanderaient $\left[\right.$ sic] qu'à s'exprimer ${ }^{\mathrm{r} 6}{ }^{\prime}$. Le choix de la correspondance comme genre littéraire de prédilection n'est pas

I4 Entrevue avec Stéphanie Boulay, Eastman, II août 2018.

is Les sociologues de la littérature Nathalie Heinich, Bernard Lahire et Gisèle Sapiro considèrent que la publication auprès d'un éditeur reconnu constitue l'une des principales "preuves sociales d'une existence en tant qu'écrivain" (Bernard Lahire, La condition littéraire, p. 213). Les écrivains amateurs ont accès à la publication, mais souvent par le recours à des procédés dévalorisés dans le champ littéraire, comme l'autoédition, ce qui ne leur octroie aucune reconnaissance de la part de l'institution. Voir Nathalie Heinich, Être écrivain. Création et identité, Paris, La Découverte, 2000, p. 68; Bernard Lahire, La condition littéraire. La double vie des écrivains, Paris, Éditions la découverte, 2006, p. 2II-2I3; Gisèle Sapiro, «Devenir écrivain.e: de la reconnaissance symbolique à la reconnaissance professionnelle», dans Gisèle Sapiro et Cécile Rabot (dir.), Profession? Écrivain, Paris, Centre national de la recherche scientifique, 2017, p. 44.

i6 Claude F. Poliak, Aux frontières du champ littéraire. Sociologie des écrivains amateurs, Paris, Economica, coll. "Études Sociologiques», 2006, p. 53. 
anodin: la forme épistolaire est à la portée de tous et peut remplir une visée à la fois artistique et utilitaire, ce qui facilite un premier contact avec la création littéraire.

L'écriture de lettres dans les jardins, contrairement aux ateliers d'écriture, n'est pas dirigée et se fait de façon solitaire. Le festivalier dispose donc d'une grande liberté lors de ces séances, qui s'étendent sur toute la durée du festival, les jardins étant ouverts du jeudi au dimanche entre Io $\mathrm{h}$ et $\mathrm{I} 7 \mathrm{~h}$. Dans son étude sur les écrivains amateurs $^{17}$, Claude F. Poliak souligne que la liberté constitue une composante essentielle de la pratique d'écriture dilettante, car la majorité des écrivains amateurs avouent écrire de façon tout à fait spontanée lorsque l'inspiration les frappe ${ }^{\mathrm{I}}$. De plus, un certain nombre d'écrivains non-initiés questionnés par Poliak font un lien entre la nature et l'inspiration. En ce sens, le milieu des jardins tend à influencer la pratique d'écriture des visiteurs. Comme ils sont amenés à écrire dans un environnement particulier, dans lequel ils sont souvent entourés d'inconnus, les festivaliers expérimentent la "création en situation ${ }^{19}$ " lorsqu'ils s'installent dans les jardins.

Le concept de la création en situation, théorisé par David Ruffel sous l'appellation de la «littérature contextuelle», met en évidence les changements affectant depuis quelques années la création littéraire, qui s'opère de moins en moins de façon solitaire. En effet, «la réactivité au contexte et la participation collective ${ }^{20}$ " prennent de plus en plus de place dans les processus de création des écrivains et des artistes d'aujourd'hui, qui placent au cour de leur pratique leurs rapports avec les autres et avec leur environnement. En ce sens, la création littéraire ne se limite plus au simple produit fini, soit le livre

17 L'étude de Poliak a été réalisée auprès de quelque 4500 participants d'un concours de nouvelles organisé en 1990 par France Loisirs. L'enquête est donc principalement basée sur des auteurs aspirant à la sphère de grande diffusion. Bien qu'il soit important de tenir compte des possibles décalages culturels entre la France et le Québec, les éléments relevés par Poliak peuvent s'appliquer à la plupart des écrivains amateurs québécois, comme l'a montré l'analyse de I69 notices biographiques de primo-romanciers québécois. Voir Marie-Pier Luneau, "De cueilleur de cerises à écrivain. La figure du primo-romancier sur les sites d'éditeurs au Québec», Voix et Images, vol. 43, no 3, 20I8, p. 93-III. $73 \%$ des écrivains amateurs interrogés s'adonnent à l'écriture seulement "lorsqu'ils éprouvent l'envie d'écrire». Claude F. Poliak, Aux frontières du champ littéraire, p. II5.

I9 David Ruffel, «Une littérature contextuelle», Littérature, $n^{\circ}$ I6o, décembre 20Io, p. 63.

$20 \quad$ Ibid., p. 64. 
publié, mais s'inscrit dans un processus de création plus large, qui est, à bien des égards, beaucoup plus significatif que son résultat. Cette nouvelle façon de concevoir la littérature «repens[e] le statut du créateur ${ }^{2 \mathrm{I}}$ ", qui évolue et crée en relation avec autrui, et favorise l'élargissement des pratiques littéraires. De même, la création in situ tend à modifier «leur perception [des écrivains] du geste de création et, au-delà, leur rapport à l'écrit, au monde et à $\operatorname{soi}^{22}{ }^{2}$. On peut donc dire que Les Correspondances d'Eastman participent à une redéfinition du fait littéraire: sur le site du festival, la littérature est conçue comme une expérience à la fois collective et solitaire inscrite dans un contexte spatio-temporel précis, ce qui dépasse largement le seul objet-livre. Le festival valorise avant tout le geste de l'écriture, sans égard au résultat final, étant donné qu'aucune volonté de publication ni même de diffusion dans l'espace public n'est associée à l'écriture de lettres dans les jardins, les missives étant directement acheminées à leur destinataire sans avoir été affichées ou lues publiquement.

\section{Le festivalier au cœur de la chaîne du livre}

Chaque année, le festival présente un thème différent qui oriente la programmation. Par exemple, le thème de l'édition de 20I8, "Les coulisses de la littérature", a donné lieu à des échanges non seulement sur l'écriture et la lecture, mais aussi sur les processus d'édition et les différents métiers pratiqués par les écrivains en dehors de la création littéraire. Un porte-parole est également associé à chacune des éditions. La différence entre les parcours des porte-paroles des plus récentes éditions, Dany Laferrière (2017), Stéphanie Boulay (2018) et Christian Bégin (2019), illustre une volonté de mettre en vedette autant des écrivains reconnus que de jeunes auteurs, mais surtout de rejoindre un public plus vaste en mettant de l'avant des figures connues du grand public. Au moment de son apparition au festival en août 20I8, Stéphanie Boulay n'avait publié qu'un seul roman, mais était très connue comme auteure-compositrice-interprète et membre du duo musical Les sœurs Boulay, alors que Dany Laferrière, auteur d'une trentaine de titres, bénéficiait d'une importante reconnaissance de la part de l'institution littéraire, notamment en tant que membre

2I Ibid., p. 67.

22 Cécile Rabot, «Les résidences d'écrivain.e.s entre création et médiation ", dans Gisèle Sapiro et Cécile Rabot (dir.), Profession? Écrivain, p. 299. 
de l'Académie française. Christian Bégin, pour sa part, avait publié quelques nouvelles dans des recueils collectifs ainsi que des livres de recettes produits dans la lignée de l'émission Curieux Bégin; sa figure publique était avant tout associée aux titres d'animateur et de comédien.

Comme il a lieu pendant la saison estivale, le festival se déroule dans une ambiance décontractée, autant pour les visiteurs que pour les auteurs invités. En 20I7, Dany Laferrière avait même affirmé que Les Correspondances d'Eastman "rim[aient] avec plaisir et vacances $^{23}$ ». Le milieu champêtre et éloigné des grands centres dans lequel les différentes activités se déroulent renforce l'impression de délassement propre au festival. Des espaces de repos, tels que les jardins d'écriture, qui incitent à la tranquillité et à la contemplation de la nature, sont mis à la disposition des visiteurs. Ces jardins représentent à eux seuls une attraction touristique, certains festivaliers se déplaçant à Eastman expressément pour les fréquenter. Le coin lounge, plus animé, permet aux visiteurs de manger, de boire un verre et de discuter. Nouveauté de 20I7, cet endroit d'échanges est propice aux rencontres entre les festivaliers, mais aussi aux rencontres entre les professionnels du livre et les visiteurs. Situé tout juste à côté de l'espace réservé aux séances de dédicaces, le coin lounge favorise les rapprochements entre les écrivains et le public. Les différents lieux de détente et d'échanges mis en place par le festival sont déterminants dans l'expérience des festivaliers, étant donné qu'ils instaurent un sentiment de communauté parmi l'ensemble des participants, peu importe leur statut, ce qui tend à accroître leur sentiment de vivre une expérience agréable ${ }^{24}$.

Bien qu'une sortie aux Correspondances d'Eastman soit présentée comme un moment de répit, le rôle des visiteurs au sein du festival est loin d'être passif. Plutôt que de se limiter à écouter les écrivains pendant les activités de la programmation, les festivaliers participent aux discussions, en posant des questions et en exprimant leur point de vue. Comme dans la plupart des événements littéraires, ils peuvent aussi converser avec les professionnels du livre lors de situations plus

23 Jean-François Gagnon, "Les Correspondances d'Eastman sans frontières", La Tribune, 23 mai 2017. http://www.latribune.ca/arts/les-correspondancesdeastman-sans-frontieres-536I32e20d83I4If7e4af3e8506I2329.

24 Alan Brown, «All the World's a Stage: Venues and Settings, and The Role They Play in Shaping Patterns of Art Participation ", dans Jennifer Radbourne, Hilary Glow et Katya Johanson (dir.), The Audience Experience: A Critical Analysis of Audiences in the Performing Arts, Bristol/Chicago, Intellect, 2013, p. 56. 
informelles, comme les séances de dédicaces ou les rencontres au coin lounge. Dans un tel contexte, la relation entre le lecteur et l'auteur est en quelque sorte renversée, ce qui octroie un pouvoir insoupçonné aux festivaliers. Tandis que, au moment de la lecture, le lecteur correspond au récepteur plus ou moins passif d'une ouvre produite par l'écrivain - ce dernier détenant le contrôle sur la communication -, les rencontres publiques mettent de l'avant le lecteur comme un producteur de sens, qui nourrit l'écrivain "en lui apportant des témoignages sur sa réception, en le poussant à une démarche réflexive et en l'amenant à aller encore plus loin que dans son texte écrit ${ }^{25}$ ». Contrairement aux rencontres publiques traditionnelles, qui sont circonscrites à un espace clos, Les Correspondances d'Eastman créent des occasions de rencontre fortuites entre les lecteurs et les auteurs: étant donné que le site du festival est dispersé aux quatre coins de la municipalité, «le festival organise un grand espace scénique où artistes

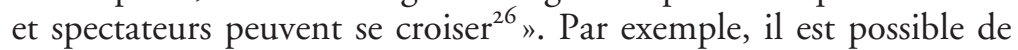
rencontrer par hasard un de ses auteurs favoris dans un commerce d'Eastman et de nouer une conversation avec lui. Pour les lecteurs, qui ont tendance à percevoir les écrivains comme des êtres inaccessibles, ce type d'expérience revêt un caractère tout à fait singulier, d'autant plus qu'il donne l'impression d'avoir accès non pas à la personnalité publique de l'auteur, comme c'est le cas lors des activités de la programmation, mais bien à sa personnalité authentique. Ce genre de rencontre, qui se déroule à l'extérieur du cadre du festival, correspond à un "unique, unplanned instant of meeting that cannot be programmed into the performance 27 ".

La proximité qui s'installe entre les écrivains professionnels et les visiteurs dans de telles situations est symptomatique de la dimension humaine et sociale au cœur de l'expérience des festivaliers. L'esprit de communauté qui fait la marque des Correspondances d'Eastman se retrouve non seulement au sein de l'organisation, à laquelle une

25 Cécile Rabot, "Donner de sa personne: les rencontres en librairie et en bibliothèque», dans Gisèle Sapiro et Cécile Rabot (dir.), Profession? Écrivain, p. 227.

26 Stéphane Carpentier, «La place du spectateur dans les arts de la rue», dans Betty Lefevre, Pascal Roland et Damien Féménias (dir.), Un festival sous le regard de ses spectateurs. Viva Cité, le public est dans la rue, p. 99.

27 «Un instant de rencontre unique et imprévu, qui ne peut être programmé au sein de la performance» [je traduis]. Rachel Gomme, «Not-So-Close Encounters: Searching for Intimacy in One-to-One Performance», Participations, vol. I2, $\mathrm{n}^{\mathrm{o}}$ I, mai 20I5, p. 297. 
grande partie du village participe, mais aussi dans les relations amicales qui s'établissent entre les auteurs et leur public. Il n'y a donc plus de réelle distinction entre les écrivains et les festivaliers, qui endossent le même statut pendant quelques heures: celui de visiteur du village d'Eastman, ou même de simple passant ${ }^{28}$. À cet égard, il est intéressant de souligner que Stéphanie Boulay, qui a assisté à plusieurs activités en tant que spectatrice, se percevait elle-même comme une festivalière en 2018, alors qu'elle était porte-parole de l'événement. On observe en ce sens que les rôles sont loin d'être figés, les auteurs empruntant par moments des caractéristiques accolées à l'activité de festivalier et vice-versa.

\section{Écrivains, les festivaliers?}

Quelle que soit l'activité à laquelle ils prennent part, les visiteurs demeurent actifs, même lorsqu'ils sont silencieux. Soulignons d'emblée que le silence ne reflète pas forcément un état d'inactivité chez les spectateurs. Selon Jacques Rancière, l'association entre le regard, l'écoute et la passivité ne va pas de soi. Au contraire, l'écoute et le regard traduisent bien souvent une activité intellectuelle et cognitive, les spectateurs agissant comme "des interprètes actifs du spectacle qui leur est proposé29". Le fait d'écouter attentivement les auteurs dans un lieu dédié à la réflexion intellectuelle implique un acte de réception et d'interprétation complexe chez les festivaliers, en plus d'instaurer le sentiment d'une complicité entre les écrivains et le public, qui sont investis dans une forme de "communal dialogue" 30 ". Sur le site des Correspondances d'Eastman, particulièrement lors des ateliers d'écriture, mais aussi lors des entrevues et des tables rondes, il n'est pas rare de voir les spectateurs munis de leur carnet de notes. Deux jeunes filles, rêvant de devenir romancières, prenaient même des notes pendant une activité jeunesse animée par Sonia Sarfati, en 2019. La qualité d'écoute exceptionnelle du public pendant les lectures publiques, les entrevues et les cafés littéraires a été soulignée par Étienne Beaulieu, directeur de la programmation, lors de la

28 Gisèle Sapiro (dir.), L'écrivain.e à la rencontre de son public. Enquête sur le public du festival Les Correspondances de Manosque, p. 24.

29 Jacques Rancière, Le spectateur émancipé, Paris, La fabrique éditions, 2008, p. I9.

30 " "dialogue communautaire" " [je traduis]. Katya Johanson et Robin Freeman, "The reader as audience: The appeal of the writers' festival to the contemporary audience», Continuum, vol. 26, $\mathrm{n}^{\circ}$ 2, avril 20I2, p. 3II. 
cérémonie de clôture de la I6 édition du festival, en 20I8. De même, plusieurs auteurs et animateurs présents à la $17^{\mathrm{e}}$ édition, tels que Biz et Christian Bégin, se sont dit surpris par l' «écoute palpable ${ }^{31}$ » dont le public faisait preuve, ce qui s'observe rarement dans une assistance aussi nombreuse.

Les festivaliers sont d'autant plus actifs lorsqu'ils écrivent euxmêmes des textes, que ce soit de façon accompagnée, pendant les ateliers de création, ou de façon solitaire, dans les jardins d'écriture. En dehors de ces deux activités, le festival encourage spécialement la création littéraire par la tenue de deux concours d'écriture, dont le premier s'adresse aux élèves des écoles primaires de la région d'Eastman. En juin, à la fin de l'année scolaire, un auteur associé au volet jeunesse des Correspondances d'Eastman se déplace dans les classes afin de donner des ateliers de création spécialement conçus pour le concours. À l'aide d'un écrivain professionnel, qui les guide dans ce processus, les élèves sont amenés à prendre la plume afin de rédiger un court texte épistolaire. Les gagnants sont dévoilés lors de la cérémonie d'ouverture du festival, pendant laquelle l'écrivain ayant chapeauté les ateliers d'écriture procède à la lecture des lettres lauréates. Les apprentis écrivains sont ensuite invités à l'avant pour recevoir leur prix.

Le deuxième concours d'écriture, au départ nommé "Concours de la Poste restante" puis rebaptisé "Concours de l'interlettre» en 2013, est de plus grande envergure puisqu'il est ouvert à tous. Les participants de ce concours n'ont pas besoin d'être physiquement présents au festival pour prendre part à l'activité. Il est tout de même intéressant de se pencher sur la façon dont ils sont traités dans le cadre des Correspondances d'Eastman, par rapport aux écrivains invités. Contrairement à ces derniers, une fois leur texte écrit et envoyé, les participants du concours ne jouent pas un rôle de premier plan au sein du festival. Seuls les finalistes disposent d'une certaine visibilité pendant les festivités, étant donné que leur création est lue par des comédiens professionnels lors de la cérémonie de clôture. Quoiqu'ils soient invités sur scène après les lectures, les écrivains amateurs primés sont présents uniquement à titre de spectateurs, et non à titre d'auteurs invités. Il est à noter que certains finalistes ne se déplacent même pas sur le site du festival et n'assistent pas à la cérémonie de clôture. Les auteurs des lettres retenues par le jury reçoivent «des livres dédicacés

31 Allocution de Biz (futur porte-parole de la I8 édition), cérémonie de clôture, $\mathrm{I} 7^{\mathrm{e}}$ édition du festival Les Correspondances d'Eastman, II août 2019. 
des membres du jury, de petits cadeaux, leurs lettres sont lues devant public lors de la cérémonie de clôture des Correspondances et elles sont affichées ci-dessous [sur le site web du festival] ${ }^{32}$ ». Un prix de $500 \$$ est remis au meilleur texte, dont la version audio est diffusée sur la plateforme web La Fabrique culturelle. Les finalistes bénéficient donc d'une reconnaissance symbolique de la part du festival, à laquelle s'ajoute une reconnaissance financière pour le gagnant.

En proposant ces deux concours de création littéraire, Les Correspondances d'Eastman récompensent le travail des écrivains amateurs de toutes les générations et encouragent leur reconnaissance, en plus de leur offrir une plateforme de diffusion. Le festival s'érige en ce sens comme une instance de consécration "en simili ${ }^{33}$ ", c'est-à-dire une instance plus ou moins externe au champ littéraire qui s'octroie le pouvoir d'accorder à des auteurs amateurs le titre d'écrivain. Cela dit, la plateforme de diffusion offerte aux écrivains primés s'inscrit "aux frontières du champ littéraire ${ }^{34}$ ", étant donné qu'elle s'éloigne du moyen de diffusion traditionnel du milieu littéraire, c'est-à-dire la publication sous forme de livre auprès d'un éditeur reconnu, qui concrétise l'identité d'écrivain ${ }^{35}$. La diffusion des textes via une plateforme web, et non par le biais d'une maison d'édition, souligne la distinction entre les lauréats du concours et les professionnels invités au festival, qui ont tous publié au moins un titre chez un éditeur littéraire établi.

L'intérêt montré par l'organisation envers les écrivains amateurs ne suffit pas à abolir la hiérarchie entre les amateurs et les professionnels, étant donné que le traitement réservé aux auteurs invités tend à les distinguer de la masse des festivaliers; les visiteurs se déplacent et paient un prix d'entrée ${ }^{36}$ pour les entendre parler de leurs expériences. Dans le cadre d'un événement mettant en vedette des professionnels de l'écriture, l'argent agit comme un «symbole des rapports sociaux

32 Les Correspondances d'Eastman. http://www.lescorrespondances.ca/archives/ coups-de-coeur-20I8.

33 Claude F. Poliak, Aux frontières du champ littéraire. Sociologie des écrivains amateurs, p. 52.

34 Ibid., p. 221.

35 Nathalie Heinich, Etre écrivain. Création et identité, $367 \mathrm{p}$.

36 En 2019, le prix d'entrée pour les cafés littéraires et les grandes entrevues, d'une durée approximative d'une heure, était de $24 \$$ pour les clients réguliers et de I2 \$ pour les étudiants. L'ensemble des activités jeunesse étaient gratuites, et les lectures publiques et les ateliers d'écriture étaient accessibles avec le stylopasseport, vendu $14 \$$. 
qui par sa médiation s'instituent ${ }^{37} »$. Les rapports entre celui qui paie et celui qui est payé sont nécessairement hiérarchisés, ce qui accentue la distinction entre auteurs et spectateurs. La présence des festivaliers sous le chapiteau renforce à elle seule le statut distinct dont bénéficient les écrivains professionnels. Selon une bénévole rencontrée en 20I7, les auteurs aiment particulièrement être invités aux Correspondances d'Eastman parce qu'ils y sont reçus en tant qu'écrivains, et entièrement reconnus comme tels, notamment dans les rencontres où ils sont principalement questionnés sur leurs œuvres et sur leur pratique d'écriture. Une invitation au festival confirmerait ainsi leur statut d'écrivain: "mis.e en scène comme écrivain.e, il ou elle est auteur.e dans le regard des autres, ce qui l'autorise à se voir et à se dire luimême ou elle-même auteur. $e^{38}$." Lors des activités, une distance symbolique s'ajoute à la distance physique entre celui qui parle, l'écrivain professionnel, et celui qui écoute, le festivalier. En plus de se distinguer physiquement de la masse de spectateurs par leur occupation de l'espace, les écrivains professionnels se différencient par leur statut professionnel. La déférence dont les organisateurs et les festivaliers font preuve à leur égard contribue en outre à magnifier leur statut au sein du festival. À cet égard, le directeur général de l'événement considère qu'il est primordial d'accueillir les écrivains comme de véritables artistes professionnels en leur offrant un cachet substantiel et en défrayant leur hébergement, leurs repas et leurs déplacements ${ }^{39}$.

\section{Proximité et distance entre le festivalier et l'écrivain}

L'activité des brunchs littéraires, à laquelle seule une trentaine de personnes assiste dans un bistro exigu, illustre bien la complexité des rapports entre les festivaliers et les auteurs, qui tendent à être à la fois rapprochés et éloignés sur le site des Correspondances d'Eastman. Les brunchs littéraires mettent en vedette un écrivain d'assez grande notoriété, par exemple Michel Rabagliati (2017), Kim Thúy (2018) ou Joséphine Bacon (2019), qui discourt sur son parcours d'auteur

37 Damien Féménias, «Publiciser la culture en démocratie: la gratuité, un rêve de société?», dans Betty Lefevre, Pascal Roland et Damien Féménias (dir.), Un festival sous le regard de ses spectateurs. Viva Cité, le public est dans la rue, p. 207.

38 Cécile Rabot, "Les résidences d'écrivain.e.s entre création et médiation", dans Gisèle Sapiro et Cécile Rabot (dir.), Profession? Écrivain, p. 293-294.

39 Entrevue avec Raphaël Bédard-Chartrand, Eastman, II septembre 2017. 
pendant que les spectateurs déjeunent. La configuration du local, dans lequel les spectateurs disposent de peu d'espace, provoque dès le départ "a closer relationship with those around that will cause reactions to a performance to be magnified ${ }^{40}$ ", faisant naitre chez les festivaliers le sentiment de participer à une expérience unique. L'étroitesse de la salle et le nombre restreint de spectateurs instaurent une intimité qui semble exacerbée par le ton de la confession employé par l'écrivain. Cette atmosphère de proximité favorise les interventions spontanées des membres du public, durant l'activité, et les discussions amicales lors de la période de questions. Une fois le brunch terminé, certains festivaliers vont même jusqu'à témoigner leur affection envers l'écrivain invité par le biais de bises et d'embrassades ${ }^{4 \mathrm{I}}$, les contacts physiques agissant de façon à abolir toute distance, du moins physique, entre les deux parties. L'écrivain se distingue du lot par la position qu'il occupe dans l'espace, étant donné qu'il est placé à l'avant. Son statut de personnalité publique — on peut même, dans certains cas, parler d'un statut de célébrité, comme pour Kim Thúy, qui est bien connue à l'extérieur du cercle des adeptes de littérature ${ }^{42}$ — tend à instaurer une distance symbolique additionnelle entre l'écrivain et le public.

Lors de la séance de questions du brunch littéraire avec Dominique Demers, le samedi II août 20I8, les festivaliers se sont surtout intéressés à la démarche d'écriture de l'auteure, la plupart souhaitant recevoir des conseils pour nourrir leur propre pratique. Le fait que la plupart des festivaliers se prêtent eux-mêmes à la création littéraire, de façon amatrice ou professionnelle, expliquerait "why so many questions to authors at festivals are "how to" questions, asking them to describe their techniques, work habits and dealings with agents and publishers ${ }^{43}$ ". Dans cette optique, les écrivains invités se démarquent nettement des spectateurs grâce à leur expérience de l'écriture et du métier d'écrivain, dont ils font bénéficier l'auditoire. On peut donc dire que l'activité du brunch littéraire instaure une proximité entre l'écrivain et son

40 «Une relation plus proche avec ceux qui les entourent, faisant en sorte que les réactions à la performance seront amplifiées» [je traduis]. Gareth White, Audience Participation in Theatre: Aesthetics of the Invitation, New York, Palgrave Macmillan, 2013, p. 136.

4I Voir la photo en Annexe IV.

42 À titre d'exemple, la page Facebook de l'auteure est suivie par plus de I6 ooo abonnés.

43 "Pourquoi autant de questions dans les festivals littéraires sont des questions sur le "comment", interrogeant les auteurs sur leurs techniques d'écriture, leurs habitudes de travail et leurs relations avec les agents et les éditeurs» [je traduis]. Wenche Ommundsen, «Literary Festivals and Cultural Consumption», p. 28. 
public, tout en mettant le premier sur un piédestal. À cet égard, les festivaliers jouent un rôle important dans cette distanciation, étant donné que leur façon de se comporter tend à accorder une certaine supériorité aux écrivains invités. Le fait de demander des conseils d'écriture, par exemple, instaure un rapport semblable à celui de l'élève vis-à-vis de son maître.

Lorsqu'ils animent des ateliers de création, les écrivains endossent un rôle similaire à celui d'un professeur, puisque les ateliers tendent à reproduire les codes pédagogiques propres au milieu scolaire. La dynamique particulière qui s'installe entre le pédagogue et l'apprenant accroît la distinction entre les auteurs et les festivaliers, les premiers étant «investi[s] par le groupe d'un statut spécial, d'une "aura" 44 ». Ce rapport hiérarchique qui s'observe entre le maître et l'apprenti differe selon la façon dont l'atelier est orchestré par l'écrivain, mais aussi selon le lieu dans lequel il se déroule et la façon dont celui-ci est occupé. En cas de pluie, l'activité a lieu dans une salle communautaire, dans laquelle des tables sont disposées soit en cercle, soit en rangée, selon les cas. Si l'espace est aménagé comme une salle de classe ${ }^{45}$, l'impression d'être à l'école, en situation d'apprentissage, sera renforcée. Dès lors, le partage du savoir sera davantage unidirectionnel, allant du maitre vers l'élève. Or, le simple fait de placer les tables en cercle instaure une certaine convivialité au cour de l'atelier et amoindrit les hiérarchies entre l'écrivain professionnel et les écrivains amateurs, si bien que l'animateur se fond parfois dans la masse de participants, surtout s'il participe lui-même aux exercices proposés ${ }^{46}$.

Lorsque la température est clémente, les ateliers de création ont lieu en plein air, dans les jardins d'écriture. Cet espace informel favorise les échanges amicaux et rend les barrières entre l'auteur établi et les amateurs plus perméables. La forêt, et plus largement la nature, instaure un sentiment de communion et de convivialité parmi les participants: l'espace du bois est si éloigné «du cadre de l'art "académique" " qu'il est considéré par les festivaliers comme "plus permissif $\left[\ldots\right.$ et] plus démocratique ${ }^{47}$ " que l'espace urbain. Le partage d'un tel espace facilite l'établissement d'un climat de

44 André Marquis et Hélène Guy, L'atelier d'écriture en questions. Du désir d'écrire à l'élaboration du récit, Montréal, Éditions Alias, coll. "Alias poche», 2007, p. 29.

45 Voir la photo en Annexe V.I.

46 Voir la photo en Annexe V.II.

47 Betty Lefevre, "Le Bois réenchanté», dans Betty Lefevre, Pascal Roland et Damien Féménias (dir.), Un festival sous le regard de ses spectateurs. Viva Cité, le public est dans la rue, p. 83 . 
confiance mutuelle, ce qui renforce le sentiment pour les participants de tisser une relation significative avec l'écrivain professionnel et de faire partie du même groupe que lui ${ }^{48}$. Il demeure que l'animateur occupe un statut privilégié au sein de l'atelier, dans le sens où il en contrôle le déroulement, en plus d'être le seul à se tenir debout, au centre des participants: l'attention est tournée vers lui. De surcroît, avant de plonger dans les exercices d'écriture, l'écrivain prend le soin de se présenter et de mettre de l'avant ses réalisations dans le monde littéraire, ce qui souligne son appartenance professionnelle à un domaine qui, pour la plupart des festivaliers, se réduit à une activité de loisir. De ce fait, une distinction s'opère bel et bien entre l'écrivain, dont le travail est reconnu par l'institution littéraire, et les festivaliers, dont la pratique demeure bien souvent cantonnée aux «loisirs créatifs ${ }^{49}$ " qui, selon Tanguy Habrand, constituent un type particulier de pratiques littéraires s'inscrivant hors du circuit traditionnel de l'édition. Comportant une dimension créative et artistique, l'activité d'écriture propre aux loisirs créatifs n'aboutit toutefois pas à un projet publié. C'est donc principalement la diffusion du travail d'écriture qui sépare les deux groupes: contrairement à l'écriture des amateurs, les projets des écrivains invités circulent dans l'espace public, "auquel seul.e.s accèdent les écrivain.e.s reconnu.e.s comme tel.le. so $^{5}$.

De la même façon que les festivaliers s'improvisent écrivains pendant Les Correspondances d'Eastman, certains auteurs invités endossent le rôle de visiteurs lorsqu'ils ne se produisent pas euxmêmes devant le public. Comme les écrivains ont accès gratuitement à tous les cafés littéraires ainsi qu'aux entrevues, certains intègrent l'assistance, allant souvent jusqu'à formuler des questions pendant la période accordée aux interventions du public. Par exemple, lors de la période de questions du café littéraire "Écrire et éditer» (IO août 20I8), l'auteure et éditrice Dominique Demers a interrogé les panélistes sur les ressources disponibles pour les apprentis auteurs souhaitant publier un livre. Pendant son intervention, elle n'a pas précisé son identité ni son statut au sein du festival. Ce faisant, elle a agi en tant que simple

48 André Marquis et Hélène Guy, L'atelier d'écriture en questions. Du désir d'écrire à l'élaboration du récit, p. 29.

49 Tanguy Habrand, «L'édition hors édition : vers un modèle dynamique. Pratiques sauvages, parallèles, sécantes et proscrites", Mémoires du livre/Studies in Book Culture, [En ligne], vol. 8, $\mathrm{n}^{\mathrm{o}} \mathrm{I}$, automne 20I6. http://doi.org/IO.7202/I038028ar.

so Gisèle Sapiro (dir.), L'écrivain.e à la rencontre de son public. Enquête sur le public du festival Les Correspondances de Manosque, p. I7. 
spectatrice, et non en tant qu'écrivaine invitée, bien que plusieurs spectateurs l'aient probablement reconnue lors de sa prise de parole. Cet exemple démontre que les frontières entre le rôle d'auteur invité et celui du festivalier ne sont pas tout à fait étanches dans le cadre des Correspondances d'Eastman, et nuance le constat de Myrtille Picaud ${ }^{5 \mathrm{I}}$, selon laquelle les professionnels du livre refusent d'être considérés comme de simples spectateurs, à cause de leur statut privilégié au sein de l'événement, et s'assurent de marquer leur distance avec la masse de lecteurs. Cela dit, l'attitude adoptée par les écrivains differe selon leur propre conception de l'événement ainsi que de leur statut et de leur rôle au sein de celui-ci. L'exemple de Dominique Demers, dont le cas ne fait pas exception, met en relief les différences culturelles entre la France et le Québec en ce qui a trait à la position des écrivains dans la société, les écrivains québécois étant moins portés à vouloir se distancier du public ${ }^{52}$.

Les festivaliers et les écrivains professionnels partagent plusieurs statuts dans le cadre du festival, soit ceux de spectateur, de lecteur et d'auteur ${ }^{33}$. Cependant, que ce soit pendant un café littéraire auquel ils participent ou en dehors des activités programmées, les écrivains invités s'inscrivent durant tout leur séjour à Eastman comme des promoteurs de leur propre ouvre et de leur image professionnelle. Comme l'a montré Judith Mayer dans son étude sur Les Correspondances de Manosque, les comportements d'un écrivain sur le site du festival sont pensés dans l'optique de constituer ou d'entretenir son «image de marque ${ }^{54} »$. Qu'il soit en séance de dédicaces ou en balade dans les rues du village, un écrivain "reste le porte-parole de son œuvre pendant toute la durée de la manifestation ${ }^{55}$ ". Dès lors, le statut de l'auteur invité, même lorsqu'il se mêle à la foule en tant que spectateur, demeure foncièrement différent de celui du festivalier. Malgré l'apparence de vacances des Correspondances d'Eastman, la présence

5I Myrtille Picaud, "Définitions concurrentes et caractéristiques "du public" au festival littéraire de Manosque», Interrogations, $\mathrm{n}^{\circ}$ 24, 20I7, II p.

52 Voir Michel Biron, L'absence du maître. Saint-Denys Garneau, Ferron, Ducharme, Montréal, Les Presses de l'Université de Montréal, 2000, 320 p.; Michel Biron, La conscience du désert. Essais sur la littérature au Québec et ailleurs, coll. «Papiers collés», Montréal, Boréal, 2010, 213 p.

53 Wenche Ommundsen, «Literary Festivals and Cultural Consumption», p. 28-29.

54 Judith Mayer, «Festival et prescription littéraire: L'exemple des Correspondances de Manosque", dans Brigitte Chapelain et Sylvie Ducas (dir.), Prescription culturelle: avatars et médiamorphoses, Villeurbanne (France), Presses de l'Enssib, coll. «Papiers", 2018, p. 229.

55 Ibid., p. 229. 
d'un auteur au festival ne représente pas un simple divertissement, mais bien une partie intégrante de son métier, d'autant plus qu'une contribution financière considérable lui est remise en échange de sa participation. L'insécurité financière liée au métier d'écrivain ainsi que les nouvelles exigences du lectorat, pour qui rencontrer l'auteur a acquis une grande importance ${ }^{56}$, obligent les écrivains à sortir du strict cadre de la création littéraire et à diversifier leurs activités. En effet, ils sont eux-mêmes responsables d'une partie de la promotion de leur ouvre par le biais de différentes apparitions publiques, dont font partie les festivals littéraires.

\section{Conclusion}

Dans le cadre des Correspondances d'Eastman, les festivaliers endossent simultanément et successivement différents rôles: leur figure concentre ceux du lecteur, du spectateur et de l'auteur amateur. Leurs interventions orientent les discussions et mettent souvent en évidence leur désir de se documenter sur le métier d'écrivain. Le café littéraire "Écrire et éditer», dont il a été question plus haut, a suscité par exemple plusieurs questions et commentaires du public. La révélation du grand nombre de manuscrits refusés chaque année par les maisons d'édition a retenu l'attention des spectateurs, et plusieurs festivaliers ont questionné les éditeurs à propos de leurs critères et leur processus de sélection. Les interventions et les réactions des festivaliers apparaissent comme des facteurs déterminants par rapport aux sujets traités lors des entrevues. Pour cette raison, les festivaliers incarnent aussi, à certains moments, la figure de l'animateur. Les rapports qu'ils entretiennent avec les professionnels du livre révèlent une tension constante entre la distance et la proximité, qui s'installent tour à tour entre l'écrivain et son public au sein des Correspondances d'Eastman. De ce fait, même lorsque le festivalier se perçoit comme un écrivain, son statut demeure toujours moindre par rapport à celui des écrivains invités. Parfois considéré comme un visiteur parmi d'autres, l'écrivain n'en est pas moins un professionnel présent sur le site du festival pour partager son expérience auprès des lecteurs et des écrivains amateurs.

$\mathrm{La}$ rencontre entre les lecteurs et les professionnels du livre est loin d'être la seule dimension collective des Correspondances d'Eastman.

s6 Jan Baetens, À voix haute. Poésie et lecture publique, Bruxelles, Impressions nouvelles, 20I6, I85 p. 
Comme une grande équipe se retrouve derrière la mise sur pied de l'événement, l'organisation du festival est nécessairement marquée par le travail collectif. On pourrait même dire que Les Correspondances d'Eastman sont le résultat des efforts d'un village entier. Les citoyens d'Eastman jouent un rôle primordial dans l'existence du festival, par exemple en assistant aux activités et en s'impliquant en tant que bénévoles ${ }^{57}$. De plus, en aménageant et en prêtant leur jardin, "les habitants acceptent de voir leur espace intime investi par le public à l'occasion d'une intrusion physique et/ou symbolique ${ }^{58}$ ". Sans cette collaboration des citoyens, le festival perdrait la dimension communautaire et sociale qui semble primordiale dans l'expérience des festivaliers, ceux-ci appréciant particulièrement l'ambiance conviviale de l'événement. L'aspect collectif des Correspondances d'Eastman se perçoit aussi par l'interdisciplinarité des activités proposées. La programmation met non seulement à l'honneur la lecture et l'écriture, mais aussi des spectacles multidisciplinaires alliant la littérature, la musique et le théâtre. Quelques expositions d'arts visuels sont également accessibles pendant toute la durée du festival. La description que donne Judith Mayer du festival des Correspondances de Manosque s'applique donc parfaitement aux Correspondances d'Eastman: "Au-delà de la simple correspondance épistolaire, cet événement se conçoit comme un espace d'échanges de tous ordres: entre auteur et public, entre professionnels du secteur, entre disciplines artistiques $^{59}$." Ainsi, le travail collectif et la sociabilité sont partie prenante des Correspondances d'Eastman. Sorte de microcosme du champ littéraire ${ }^{60}$, ce festival réunit différents professionnels et facilite leur contact avec un agent souvent invisible, mais non moins essentiel à la chaîne du livre, c'est-à-dire le lecteur, qui cumule pendant quelques jours différents rôles pour incarner celui de festivalier.

57 Une centaine de bénévoles, pour la plupart résidant à Eastman, s’impliquent chaque année au festival.

58 Audrey Bottineau, «Un voyage merveilleux», dans Betty Lefevre, Pascal Roland et Damien Féménias (dir.), Un festival sous le regard de ses spectateurs. Viva Cité, le public est dans la rue, p. 125 .

59 Judith Mayer, «Festival et prescription littéraire: L'exemple des Correspondances de Manosque», dans Brigitte Chapelain et Sylvie Ducas (dir.), Prescription culturelle: avatars et médiamorphoses, p. 222.

60 Millicent Weber, Literary Festivals and Contemporary Book Culture, Cham, Palgrave MacMillan, 2018, $272 \mathrm{p}$. 
ANNEXE I: Guide d'entrevue

\section{Raphaël Bédard-Chartrand}

1. Situation sociodémographique

a. Quelles sont vos caractéristiques sociodémographiques: âge, sexe, origines familiales, lieu de résidence et de travail, etc.?

b. Quelles sont vos caractéristiques professionnelles: types de formation et niveau de scolarité, métier.s exercé.s, situation économique, etc.?

c. Comment décririez-vous vos habitudes culturelles et littéraires: fréquence et intensité des activités de lecture et d'écriture, fréquence des sorties culturelles, intérêt envers les événements culturels, formations artistiques et/ou littéraires, etc.?

\section{Expérience du festival}

Pouvez-vous me parler des sujets suivants?

a. Lignes directrices dans la création du festival (valeurs, objectifs de l'événement);

b. Processus d'organisation du festival: comment s'organisent les différentes étapes menant à la création de chaque édition (choix du thème et du porte-parole, établissement de la programmation, partage des tâches, partenariats, etc.);

c. Clientèles visées, promotion du festival, financement de l'événement;

d. Relation avec les écrivains invités (attitude des écrivains invités - comment envisagent-ils leur statut de professionnels au sein de l'événement?);

e. Relation avec le public (clients et bénévoles). 
Quelles sont vos perceptions et vos attentes relativement à:

a. Le festival en général (description globale du festival, similarités et différences avec les autres événements littéraires, comme les salons du livre et les soirées de poésie);

b. L'évolution du festival depuis sa fondation;

c. Le volet «jardins d'écriture»;

d. L'aménagement du site;

e. Les retombées de l'événement dans la communauté et plus largement dans le paysage littéraire estrien et québécois.

\section{Stéphanie Boulay}

1. Situation sociodémographique

a. Quelles sont vos caractéristiques sociodémographiques: âge, sexe, origines familiales, lieu de résidence et de travail, etc.?

b. Quelles sont vos caractéristiques professionnelles: types de formation et niveau de scolarité, métier.s exercé.s, situation économique, etc.?

c. Comment décririez-vous vos habitudes culturelles et littéraires: fréquence et intensité des activités de lecture et d'écriture, fréquence des sorties culturelles, intérêt envers les événements culturels, formations artistiques et/ou littéraires, etc.?

2. Rôle au sein du festival

a. Selon vous, quel rôle endossez-vous au sein du festival? Quelles sont les différences, à votre avis, entre le rôle d'écrivain invité et celui de porte-parole?

b. Comment vous situez-vous par rapport aux autres participants ou anciens porte-paroles du festival? Quels éléments nouveaux apportez-vous aux Correspondances d'Eastman? 
c. Que signifie, pour vous, le fait d'être invité aux Correspondances d'Eastman ou d'être nommé porte-parole du festival?

d. Croyez-vous que votre participation aux Correspondances d'Eastman entraîne certaines responsabilités?

e. Sentez-vous que vous devez adopter un comportement différent lors de vos interventions publiques en tant qu'écrivain, dans un festival spécifiquement dédié à la littérature, par rapport à vos apparitions publiques dans des événements plus généraux?

\section{Expérience du festival}

a. Quelle expérience générale avez-vous du festival : combien de fois y êtes-vous allé, pour combien d'éditions avez-vous été porte-parole, vous considérez-vous comme un.e habituée, avez-vous une relation de proximité avec les organisateurs?

b. Quelle expérience précise avez-vous eue du festival cette année: à quelle.s activité.s avez-vous participé en tant qu'écrivain invité, avez-vous assisté ou participé à des activités en tant que spectateur pendant votre séjour au festival? Si oui, lesquelles? Êtes-vous intervenu en tant que spectateur?

Quelles sont vos perceptions et vos attentes relativement à:

a. Le festival en général (description globale du festival, similarités et différences avec les autres événements littéraires comme les salons du livre et les soirées de poésie);

b. L'accueil qui vous est réservé sur le site;

c. La programmation et les activités proposées;

d. La proximité avec le public;

e. L'organisation et l'aménagement du site;

f. Le volet "création littéraire" (écriture de lettres dans les jardins, ateliers d'écriture): avez-vous fréquenté les jardins et/ou écrit des lettres? 
Pouvez-vous me parler plus en détail des sujets suivants?

a. Attraits principaux du festival;

b. Activités favorites et activités moins appréciées ou jamais visitées (en tant qu'écrivain invité, mais aussi en tant que visiteur);

c. Relation avec les spectateurs/lecteurs (discussions, dédicaces, contacts ou non avec eux);

d. Relation avec les autres participants - festivaliers, éditeurs, libraires, organisateurs, animateurs, etc. - (discussions, débats, ententes conclues sur le site). 
90 Papers of the Bibliographical Society of Canada 58

ANNEXE II: Résultats des études de provenance

\begin{tabular}{|c|c|c|c|}
\hline \multicolumn{3}{|l|}{ 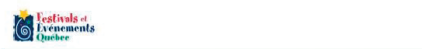 } & 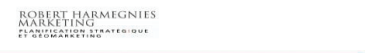 \\
\hline \multicolumn{4}{|l|}{ Présentation des résultats } \\
\hline \multicolumn{4}{|l|}{ Provenance générale } \\
\hline \multicolumn{3}{|c|}{ 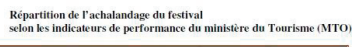 } & \multirow{3}{*}{ 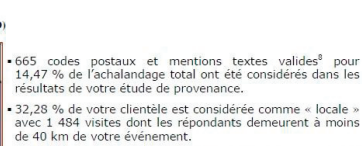 } \\
\hline \multicolumn{3}{|c|}{$\begin{array}{l}\text { Los Corrospondancos d'Eastman } \\
\text { REPAATIIION DE LACHALANDAGE } \\
\end{array}$} & \\
\hline & \begin{tabular}{|l|l} 
Nab visitios \\
2015
\end{tabular} & 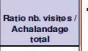 & \\
\hline 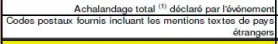 & \begin{tabular}{r|r}
4597 \\
665 \\
\end{tabular} & \begin{tabular}{|r|}
$100,00 \%$ \\
$14,47 \%$ \\
\end{tabular} & \multirow{8}{*}{ 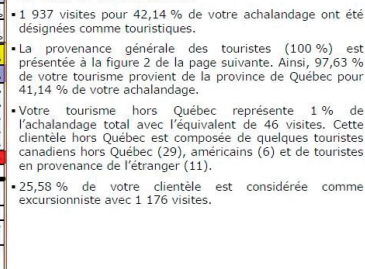 } \\
\hline Achalandage local 10 & 1484 & $32,28 \%$ & \\
\hline 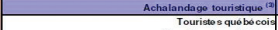 & $\frac{1937}{1691}$ & $\frac{42,14 \%}{41,14 \%}$ & \\
\hline 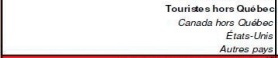 & $\begin{array}{r}46 \\
22 \\
6 \\
11 \\
\end{array}$ & $\begin{array}{l}1,00 \% \\
0.69 \% \\
0,19 \% \\
0.24 \%\end{array}$ & \\
\hline & & & \\
\hline \multirow{3}{*}{\multicolumn{3}{|c|}{ 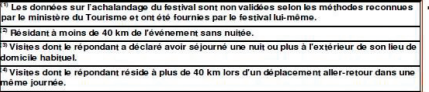 }} & \\
\hline & & & \\
\hline & & & \\
\hline \multicolumn{4}{|c|}{ 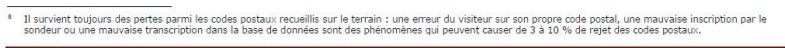 } \\
\hline \multicolumn{3}{|c|}{ 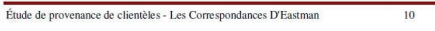 } & \\
\hline
\end{tabular}

2015

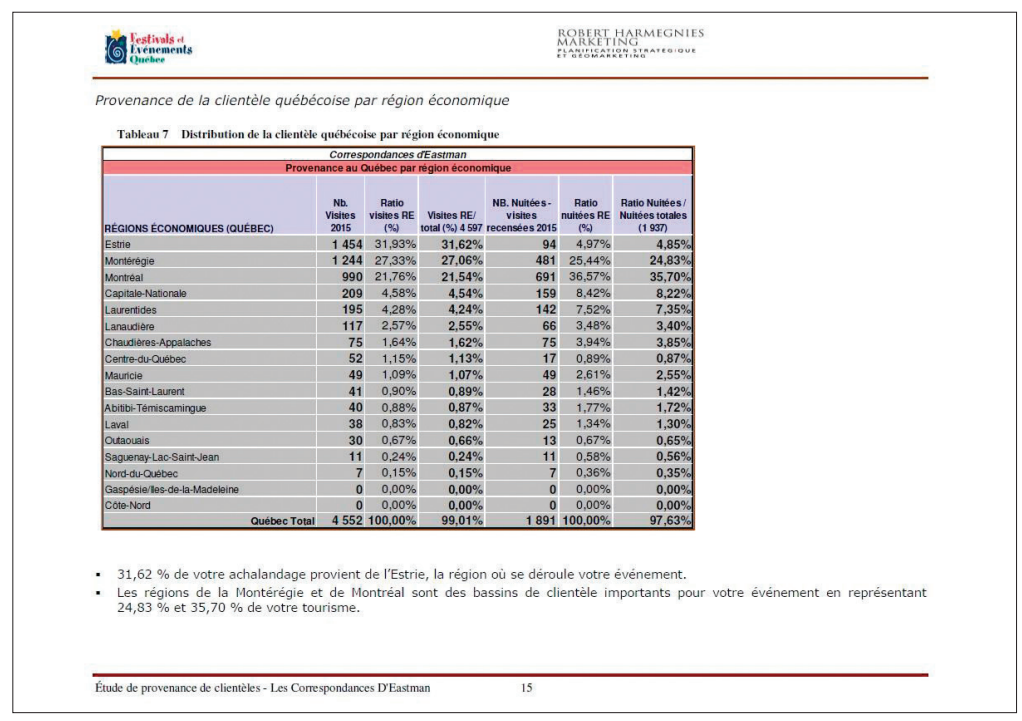




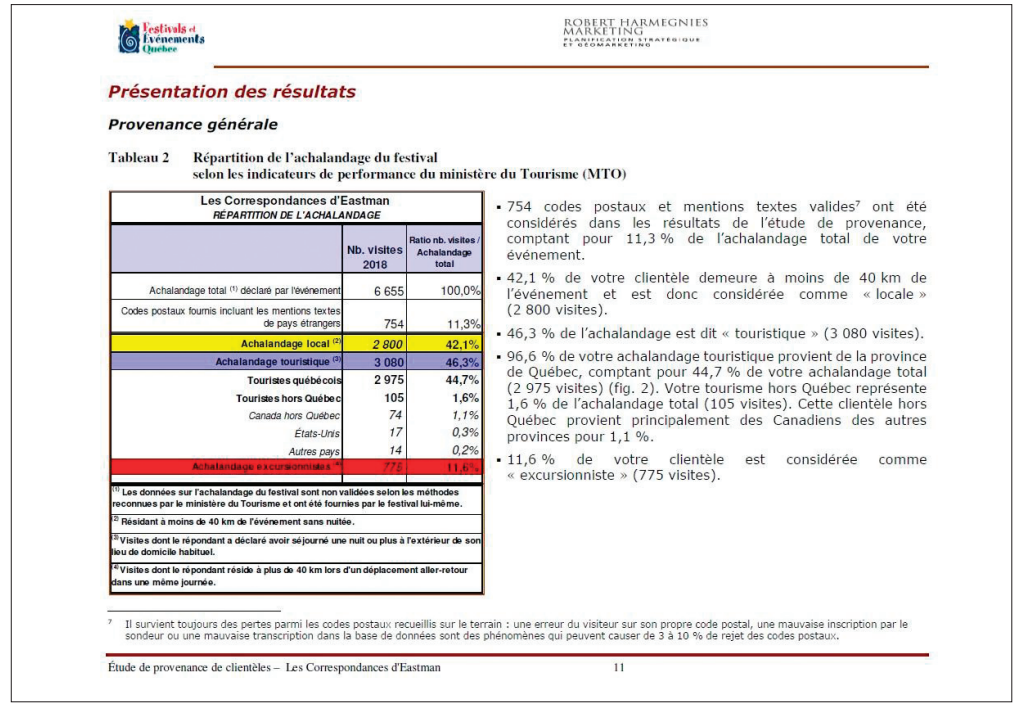

(6) Totinglents

ROBERT HARMEGNIES

Provenance de la clientèle québécoise par région économique

Tableau 7 Distribution de la clientèle québécoise par région économique

\begin{tabular}{|c|c|c|c|c|c|c|}
\hline \multirow{2}{*}{\multicolumn{7}{|c|}{ Les Correspondances d'Eastman }} \\
\hline & & & & & & \\
\hline RÉGIONS ÉCONOMIQUES (QUÉEEEC) & $\begin{array}{l}\text { Nb. Visthes } \\
2018\end{array}$ & $\begin{array}{l}\text { Ratiov vistites } \\
\text { RE (\%) }\end{array}$ & $\begin{array}{l}\text { Vistres RE total } \\
\text { (1\%)6655 } 6 \text {. }\end{array}$ & $\begin{array}{c}\text { NB. Nuiticos - } \\
\text { vistos } \\
\text { recenseges } \\
2018\end{array}$ & $\begin{array}{l}\text { Ratio nuniées } \\
\text { RE (\%) }\end{array}$ & 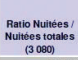 \\
\hline Estrie & 2868 & $43,8 \%$ & $43,1 \%$ & 220 & $7.4 \%$ & $7,1 \%$ \\
\hline Montreal & 1520 & $23.2 \%$ & $22,8 \%$ & 1278 & $42,9 \%$ & $41,5 \%$ \\
\hline Moneréregie & 1178 & $18,0 \%$ & $17,7 \%$ & 679 & $22,8 \%$ & $22,0 \%$ \\
\hline Capitale-Nationale & 427 & $6.5 \%$ & $6.4 \%$ & 417 & $14.0 \%$ & $13,5 \%$ \\
\hline Lanaudere & 142 & $2,2 \%$ & $2,1 \%$ & 128 & $4,3 \%$ & $4,2 \%$ \\
\hline Laurentdes & 117 & $1,8 \%$ & $1,8 \%$ & 56 & $1,9 \%$ & $1,8 \%$ \\
\hline Centre-du-Gúbec & 92 & $1,4 \%$ & $1,4 \%$ & 32 & $1,1 \%$ & $1,0 \%$ \\
\hline Mauricie & 69 & $1,0 \%$ & $1,0 \%$ & 54 & $1,8 \%$ & $1,7 \%$ \\
\hline Laval & 40 & $0,6 \%$ & $0,6 \%$ & 23 & $0,8 \%$ & $0,7 \%$ \\
\hline Chauriere-Appalacties & 39 & $0,6 \%$ & $0,6 \%$ & 39 & $1,3 \%$ & $1,3 \%$ \\
\hline Saguenay-Lac-Saint Jean & 23 & $0,4 \%$ & $0.3 \%$ & 23 & $0,8 \%$ & $0,7 \%$ \\
\hline Outaouals & 17 & $0,3 \%$ & $0,3 \%$ & 17 & $0,6 \%$ & $0,6 \%$ \\
\hline Nord-du-Cuébec & 11 & $0.2 \%$ & $0,2 \%$ & 11 & $0.4 \%$ & $0,4 \%$ \\
\hline Bas SaintLLaurent & 0 & $0,0 \%$ & $0,0 \%$ & 0 & $0,0 \%$ & $0,0 \%$ \\
\hline Gaspessie/les-de-1a-Madelaine & 0 & $0,0 \%$ & $0,0 \%$ & 0 & $0,0 \%$ & $0,0 \%$ \\
\hline Cote-Nard & 0 & $0,0 \%$ & $0,0 \%$ & 0 & $0,0 \%$ & $0,0 \%$ \\
\hline Abitibi-Temiscamingue & 0 & $0.0 \%$ & $0,0 \%$ & 0 & $0,0 \%$ & $0,0 \%$ \\
\hline Québec Total & 6545 & $100.0 \%$ & $98,3 \%$ & 2975 & $100,0 \%$ & $96.6 \%$ \\
\hline
\end{tabular}

- $43,1 \%$ de votre achalandage total provient de l'Estrie et celui-ci génère 7,1 \% de votre tourisme. 
ANNEXE III : Réponses à la question «Vous considérez-vous comme un(e) habitué(e) des événements littéraires et culturels?»

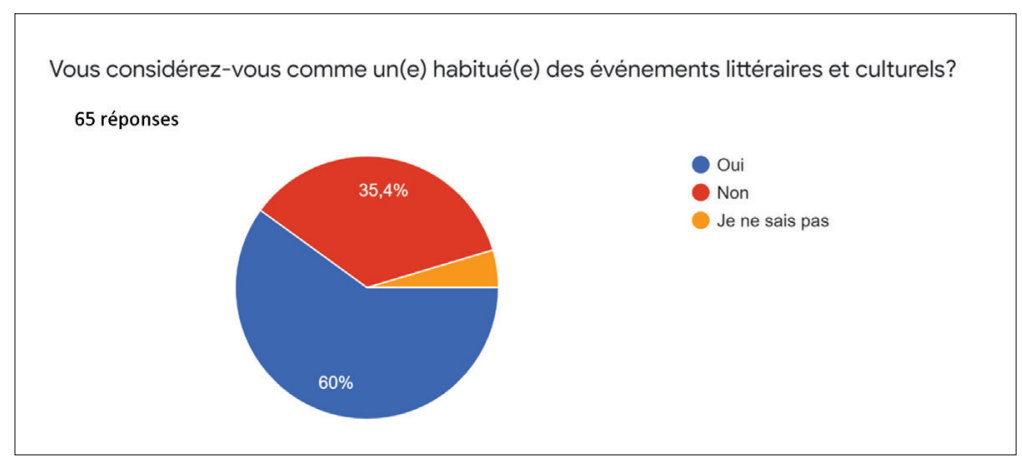

ANNEXE IV: Brunch littéraire avec Joséphine Bacon, Café Bistro les 3 Grâces, dimanche II août 2019 (photographie de Mylène Fréchette)

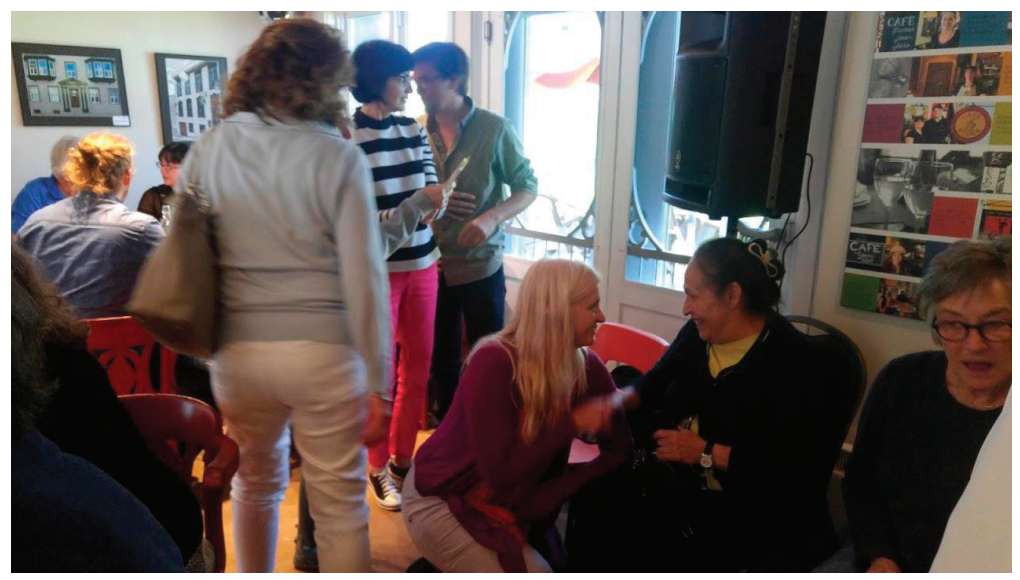


ANNEXE V.I : Atelier d'écriture avec Jason Roy, Salle Missisquoise, jeudi 9 août 2018 (photographie de Mylène Fréchette)

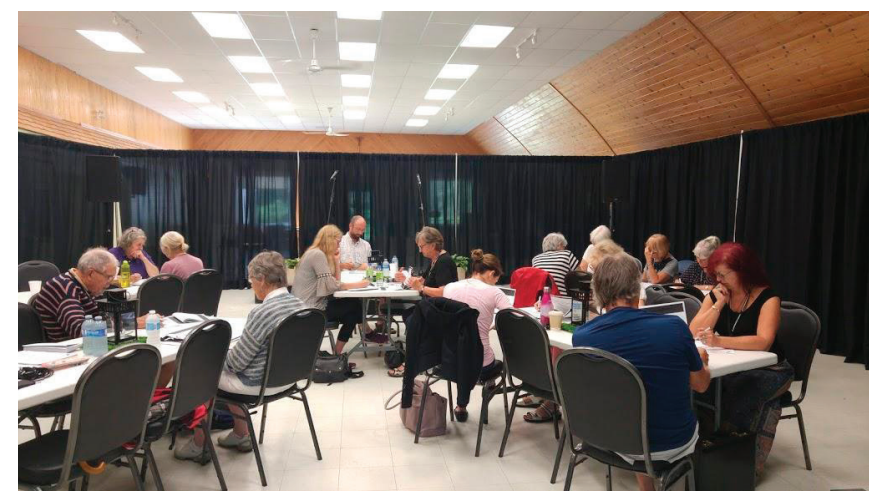

ANNEXE V.II: Atelier d'écriture avec William S. Messier, Salle Missisquoise, samedi Io août 2019 (photographie de Mylène Fréchette)

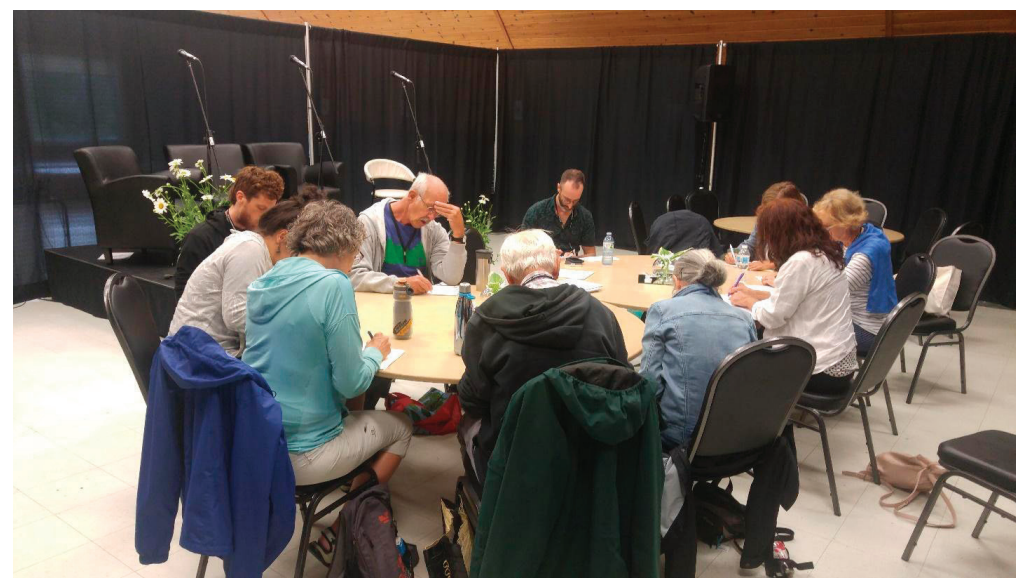




\section{Notice biographique de l'autrice}

Mylène Fréchette détient un diplôme de maittrise en études françaises de l'Université de Sherbrooke. Son mémoire portait sur le festival littéraire Les Correspondances d'Eastman. Assistante de recherches pour le Groupe de recherches et d'études sur le livre au Québec (GRÉLQ) de 2017 à 2020, elle a également été secrétaire adjointe de l'Association québécoise pour l'étude de l'imprimé (AQÉI) et corédactrice en chef de Cavale, la revue de littérature et d'arts visuels des étudiant.e.s de l'Université de Sherbrooke, de 2018 à 2020. 